\title{
Coupling of an erbium spin ensemble to a superconducting resonator
}

\author{
Matthias U. Staudt ${ }^{1}$ *Io-Chun Hoi ${ }^{1}$, Philip Krantz ${ }^{1}$, Martin Sandberg ${ }^{1}$, Michaël Simoen ${ }^{1}$, Pavel Bushev ${ }^{2}$, Nicolas \\ Sangouard $^{3}$, Mikael Afzelius ${ }^{3}$, Vitaly S. Shumeiko ${ }^{1}$, Göran Johansson ${ }^{1}$, Per Delsing ${ }^{1}$, and C. M. Wilson ${ }^{1}$ \\ ${ }^{1}$ Microtechnology and Nanoscience, MC2, Chalmers University of Technology, SE-41296 Göteborg, Sweden \\ ${ }^{2}$ Physikalisches Institut, Karlsruhe Institute of Technology, D-76128 Karlsruhe, Germany and \\ ${ }^{3}$ Group of Applied Physics, University of Geneva, CH-1211 Geneva 4, Switzerland
}

(Dated: August 30, 2018)

\begin{abstract}
A quantum coherent interface between optical and microwave photons can be used as a basic building block within a future quantum information network. The interface is envisioned as an ensemble of rare-earth ions coupled to a superconducting resonator, allowing for coherent transfer between optical and microwave photons. Towards this end, we have realized a hybrid device coupling a $\mathrm{Er}^{3+}$ doped $\mathrm{Y}_{2} \mathrm{SiO}_{5}$ crystal in a superconducting coplanar waveguide cavity. We observe a collective spin coupling of $4 \mathrm{MHz}$ and a spin linewdith of down to $75 \mathrm{MHz}$.
\end{abstract}

\section{INTRODUCTION}

The boundary set by the largest possible size of an individual quantum processing unit can be surpassed by a quantum network ${ }^{1}$. Thus quantum networks may be crucial for the applications of quantum computing, communication and metrology 2 4. In such networks, quantum information is distributed through the network and processed in nodes ${ }^{5}$. Entanglement is distributed via the channels, where optical photons carry the quantum information. The nodes can contain ensembles of real or artificial ions or atoms. Superconducting qubit $\$ \frac{6}{6}$ are promising solid-state candidates for such nodes. As superconducting qubits work in the microwave range $(\mathrm{GHz})$ and optical photons are typically in the telecom frequency (200 THz), a coherent quantum interface that can bridge the gap between these energy regimes is needed. This would lead to the combination of two fruitful research areas: quantum communication using optical photons and quantum processing using superconducting quantum circuits.

Strong coupling of a superconducting qubit to a highquality superconducting transmission line resonator has been demonstrated ${ }^{6}$, allowing for cavity quantum electrodynamic experiments on a chip (circuit QED). This achievement has enabled a number of quantum optics experiments using superconducting circuits \$ $^{7 / 2}$. For instance, the dynamical Casimir effect $\frac{13}{13}$, number-resolving photon detection $\frac{14}{14}$, single-photon generation $\frac{15}{15}$, twoqubit coupling via the circuit cavity ${ }^{16}$ and three - qubit entanglement $t^{[17 \mid 18}$ have been demonstrated. Recently superconducting qubits having coherence times on the order of several tens of $\mu$ s have been realized $\frac{19}{}$.

Hybrid proposals $\sqrt{20}$ aim at integrating various types of quantum systems in a circuit QED setting, for instance, atomic ensembles that would interact with the microwave resonator via electric dipole transitions (e.g. Rydberg atoms or polar molecules ${ }^{24}$ ) or via magnetic spin transitions (alkali atoms, silicon spins). The latter are particularly challenging since the magnetic dipole transitions are very weak. Even in a strongly confined resonator volume, the single-spin coupling rate would be far from sufficient, typically $g_{c} / 2 \pi \sim 10-100 \mathrm{~Hz}{ }^{25}$. The coupling can be significantly increased $\frac{20,23}{2 i a}$ a collective interaction with a spin ensemble, where the collective coupling scales as $g_{\text {coll }}=\sqrt{N} g_{c}$ with $N$ the number of atoms. This approach has recently attracted much attention and led to experimental realizations coupling superconducting resonators 26 to nitrogen-vacancy centers and $\mathrm{Er}^{3+}$ ions.

In this paper, we investigate experimentally the coupling between an ensemble of $\mathrm{Er}^{3+}$ ions doped into a $\mathrm{Y}_{2} \mathrm{SiO}_{5}$ host crystal and a superconducting coplanar waveguide resonator (CPWR) at $\mathrm{mK}$ temperatures. For a future optical to microwave interface, $\mathrm{Er}^{3+}$ ions are attractive because of the ${ }^{4} I_{15 / 2} \rightarrow{ }^{4} I_{13 / 2}$ transition around $1540 \mathrm{~nm}$, which is the so-called telecom window where absorption losses are minimal in optical fibers. Its strong first-order Zeeman effect allows for easy tuning of the spin resonance to the $\sim 5 \mathrm{GHz}$ operation frequency of circuit QED experiments. This alleviates the need for strong magnetic fields, which are incompatible with superconductivity. Furthermore, an optical quantum memory has recently already been demonstrated ${ }^{31}$ at this wavelength.

The $\mathrm{Er}^{3+}$ ion replaces $\mathrm{Y}^{3+}$ ions in the in the $\mathrm{Y}_{2} \mathrm{SiO}_{5}$ host crystal, which exists in two crystallographically inequivalent sites having C1 symmetry (hereafter named sites 1 and 2). In this work, we investigate spectroscopically both sites down to temperatures of $50 \mathrm{mK}$. Recently, magnetic coupling of $\mathrm{Er}^{3+}$ ions to a superconducting cavity has been shown in a parallel work for one occupied site and down to a temperature of $280 \mathrm{mK}^{30}$. To the best of our knowledge, the spectroscopic measurements presented here are done at the lowest temperature rare-earth ions have been spectroscopically investigated in a crystalline host matrix, though temperatures of lower than $100 \mathrm{mK}$ have investigated already in an amorphous host matrix ${ }^{32133}$.

\section{THEORY}

Rare-earth (RE) ions doped into inorganic crystals are interesting quantum systems owing to their long optical and spin coherence times ${ }^{34} 36$. They have recently been 
considered as qubits for quantum computing $37 / 38$ and as quantum memories 39 for optical photons in the context of quantum repeaters for long-distance communication.

RE ions can be divided into two groups; non-Kramers and Kramers ions, with an even and odd number of $4 f$ electrons, respectively. For the former type, the interaction with the typical low-symmetry crystal environment usually produce a closely spaced (10 - $100 \mathrm{MHz}$ ) hyperfine manifold in the ground state. As a result, the nonKramers ions generally do not have resonances appropriate for circuit QED experiments. The Kramers ions, e.g. Erbium, on the other hand, are, in low-symmetry environments left, with two degenerate electronic spin levels resulting in effective spin $S=1 / 2$ systems, named Kramers doublets. The magnetic moment of these Zeeman states can, for a given direction of the magnetic field, be expressed as $\mu_{m}=g \mu_{b}$ where the angle - dependent $g$ - factor is typically in the range 1-10 and $\mu_{b}$ is the Bohr magneton, corresponding to magnetic tuning factors of 14-140 GHz/Tesla. Therefore a sub-Tesla magnetic field would generally be sufficient to tune the spin transition into resonance with the CPWR. This approach is chosen here and has the advantage that RE spin ensembles have been studied in this frequency range for decades using electron paramagnetic resonance (EPR) experiments44.

The coupling rate of a single spin to the CPWR cavity mode is given by ${ }^{21} g_{c}=\mu_{m} \sqrt{\mu_{0} \omega_{r} /\left(2 \hbar V_{c}\right)}$, where $V_{c}$ denotes the cavity-mode volume, $\omega_{r}$ the cavity frequency and $\mu_{0}$ the vacuum permeability. This results in an ion coupling rate of the order of $\mathrm{Hz}$ for a typical electron spin magnetic moment. The single spin-CPWR coupling is thus insufficient to overcome the dissipation rate $\kappa \sim 0.1-1 \mathrm{MHz}$ typical for a CPWR. The strong coupling regime can, however, be reached via an enhanced collective coupling of a spin ensemble to the $\mathrm{CPWR}^{21|26| 29}$ : $g_{\text {coll }}=g_{c} \sqrt{N}$. The range in the number of spins $N$ for reaching strong collective coupling, $i$. e. : $g_{\text {coll }}>\kappa$, is of the order $N>\kappa^{2} / g_{c}^{2} \sim 10^{6}-10^{10}$. Diniz ${ }^{45}$ et al. showed recently that increasing the collective coupling suppresses the effects of inhomogeneous broadening, as long as the emitters spectral distribution decreases quicker than $\omega^{-2}$.

The hyperfine interaction in the ${ }^{4} I_{15 / 2}$ ground-state has been studied by O. Guillot-Noël et al. using EPR ${ }^{44}$. For the even isotopes ${ }^{162,164,166,168,170} \mathrm{Er}^{3+}$ the interaction can be described by a spin Hamiltonian consisting of a single Zeeman interaction term:

$$
H=\mu_{b} \mathbf{B} \cdot \mathbf{g} \cdot \mathbf{S},
$$

while in the case of the only odd isotope ${ }^{167} \mathrm{Er}^{3+}$ hyperfine and quadrupole interaction terms must be added:

$$
H=\mu_{b} \mathbf{B} \cdot \mathbf{g} \cdot \mathbf{S}+\mathbf{I} \cdot \mathbf{A} \cdot \mathbf{S}+\mathbf{I} \cdot \mathbf{Q} \cdot \mathbf{I}
$$

Here $\mathbf{B}$ denotes the external magnetic field, $\mathbf{S}$ the electronic spin and $\mathbf{I}$ the nuclear spin. The matrices $\mathbf{g}, \mathbf{A}$ and $\mathbf{Q}$ are the matrices describing the electronic Zeeman, hyperfine and quadrupole interactions. We neglect the weaker nuclear Zeeman effect. These are generally anisotropic due to the interaction with the surrounding crystal environment. Due to the low-symmetry sites in $\mathrm{Y}_{2} \mathrm{SiO}_{5}$, this leads to very strong spin mixing, resulting in weaker transition rules for the spin transitions in ${ }^{167} \mathrm{Er}^{3+}$.

The $\mathrm{Er}^{3+}$ ions occupy two crystallographically inequivalent sites in the $\mathrm{Y}_{2} \mathrm{SiO}_{5}$ host crystal. Each of these sites has two magnetically inequivalent subclasses that are related by $\mathrm{C} 2$ symmetry. Thus, depending on the magnetic field orientation, up to four Zeeman transitions are expected for the even isotopes 46 .

\section{EXPERIMENTAL SETUP}

We couple the $\mathrm{Er}^{3+}$ ions to a $\lambda / 2 \mathrm{CPWR}$, having a $\mathrm{Q}$ value of 568. The CPWR is realized by a $120 \mathrm{~nm}$ film of Niobium sputtered on a silicon substrate $(380 \mu \mathrm{m})$ having a center strip with a width of $100 \mu \mathrm{m}$ and gaps of $70 \mu \mathrm{m}$ to the ground planes.

Integration of the RE-doped crystal is done by placing the crystal directly on the superconducting cavity with pressure applied by a teflon frame. The cavity has its resonance frequency at $4.4 \mathrm{GHz}$. The crystal is doped with $0.02 \% \mathrm{Er}^{3+}$ ions.

We have measured the sample down to $\sim 50 \mathrm{mK}$ in a dilution refrigerator equipped with a superconducting magnet. The external magnetic field is applied parallel to the surface of the superconducting chip and is ramped at $3.3 \mathrm{mT} / \mathrm{s}$. The crystal has a dimension of $3.5 \times 4 \times 3 \mathrm{~mm}^{3}$ and is cut along the optical extinction axes $\mathrm{D}_{1}, \mathrm{D}_{2}$ and $\mathrm{b}$. The magnetic field is applied both within the $\mathrm{b}-\mathrm{D}_{1}$ plane and the superconducting chip surface and is parallel to the b-axis. The $\mathrm{D}_{2}$-axis is perpendicular to this plane. We measure the microwave transmission coefficient, $\mathrm{S}_{21}$, which contains both magnitude and phase, as a function of magnetic field. The microwave power at the entrance of the cavity is about $-110 \mathrm{dBm}$.

\section{RESULTS}

We sweep the external magnetic field between $0 \mathrm{mT}$ and $200 \mathrm{mT}$. For each applied magnetic field we measure $\mathrm{S}_{21}$ (see Fig. 1) and extract the Full Width at Half Maximum (FWHM) of the cavity resonance peaks. The extracted FWHM is plotted as a function of magnetic field in Fig. 2 and Fig. 3. The $\mathrm{S}_{21}$ spectrum shown in Fig. 1 is for the case where the $\mathrm{Er}^{3+}$ - ions are off - resonance. When the $\mathrm{Er}^{3+}$ - ions are tuned into resonance with the cavity mode, we expect to observe an increase in the measured cavity linewidth. This is beacause the resonant ions provide a loss channel for the microwave photons. Two different regions of applied field are covered in higher resolution, one around $40 \mathrm{mT}$ (Fig. 2) and the other around $140 \mathrm{mT}$ (Fig. 3). In each magnetic field region, a pair of peaks are visible. As mentioned, 


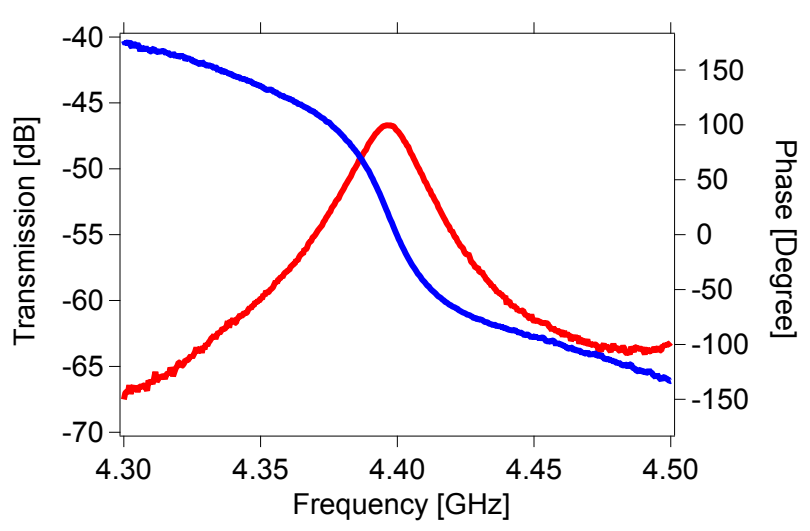

FIG. 1: Transmission spectrum of a $4.4 \mathrm{GHz}$ resonator. Both magnitude (red) and phase (blue) signal are shown. Losses in the lines to the resonator are about $90 \mathrm{~dB}$ including roomtemperature and cold attenuators. The total gain is about 40 $\mathrm{dB}$, giving rise to a maximum peak transmission of about -47 dB.

depending on the magnetic field orientation, up to four transitions are expected for Erbium, which is the case observed here. The pair of lines originating from the two crystallographic sites we denote as group 1 and 2, respectively. Lines within each pair, which are due to the magnetically inequivalent subclasses, are denoted as lines $\mathrm{a}$ and $\mathrm{b}$. We extract the g-factor from the peak position for both sites (see table 1 ). For the same crystal orientation Guillot-Noël, et al ${ }^{44}$ find a value for the g-factor of $\mathrm{g}_{1}=8.92$ and $\mathrm{g}_{2}=2.78$. The splitting for each transition and the deviation in $g$ value we attribute to a slight misalignment of the external B field to the b-axis.

If the magnetic field were perfectly aligned along the b-axis, then the subclasses would be magnetically equivalent and only two transitions visible. However, the splitting for both low and high field region are small and from the measurements in Refs. ${ }^{46} 47$ we can infer that the misalignment is of the order of $\pm 5^{\circ}$ compared to the b-axis.

We model both the spin ensemble and the cavity as a single-mode harmonic oscillator similar to Schuster et $a l . \underline{48}$. The detuning between the cavity resonance $\omega_{r}$ and atomic resonance due to the Zeeman splitting is: $\Delta_{Z}=\omega_{r}-g \mu_{b} B / \hbar$. In principal, the resonance is split due to hyperfine interaction for the ${ }^{167} \mathrm{Er}^{3+}$ ions ${ }^{44}$ (see also $\left.{ }^{49}\right)$. As the hyperfine peaks are not visible in the observed spectrum, the hyperfine interaction is not taken into account in our fit. The total width is then given as $\Gamma_{\text {tot }}=\kappa+\Gamma_{Z}$ where $\kappa$ is the cavity linewidth and $\Gamma_{Z}$ the spin induced linewidth. The spin induced linewidth is:

$$
\Gamma_{Z}=\frac{2 g_{\text {coll }}^{2} \gamma}{\left(\Delta_{Z}^{2}+\gamma^{2}\right)}
$$

where $\mathrm{g}_{c}$ is the coupling constant.

For the pair of transitions having a maximum at (a) $37.7 \mathrm{mT}$ and (b) $43 \mathrm{mT}$ (site 1) and at (a) $125 \mathrm{mT}$ and

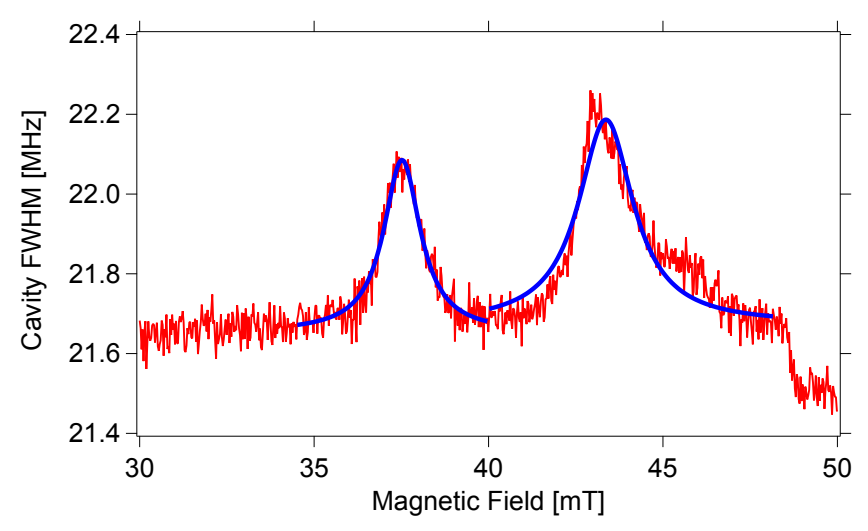

FIG. 2: Resonator linewidth as a function of magnetic field in the field region around $40 \mathrm{mT}$. Magnetic g-factors between 7 and 8 are extracted. Within each pair, the two lines represent spins aligned along different crystal axes that are related by symmetry. From the fit (solid lines, see text for details) we obtain a collective coupling rate of about $4 \mathrm{MHz}$ and a spin linewidth of about $75 \mathrm{MHz}$.

\begin{tabular}{|c||c|c|c|c|}
\hline Site & $1 \mathrm{a}$ & $1 \mathrm{~b}$ & $2 \mathrm{a}$ & $2 \mathrm{~b}$ \\
\hline $\mathrm{g}$ & 8.37 & 7.25 & 2.51 & 2.04 \\
\hline$\gamma[\mathrm{MHz}]$ & 74.9 & 96.6 & 101 & 136 \\
\hline $\mathrm{g}_{\text {coll }}[\mathrm{MHz}]$ & 4.02 & 4.98 & 6.07 & 6.16 \\
\hline
\end{tabular}

TABLE I: Measured parameters for sites 1 and 2. Here $g$ is the g-factor, $\gamma$ the spin linewidth and $g_{\text {coll }}$ the collective coupling constant.

(b) $154 \mathrm{mT}$ (site 2) we extract the parameters shown in table 1 .

In order to estimate the collective coupling constant for a temperature approaching zero, we also perform a temperature dependent measurement (see Fig. 4 for the case of site $2 \mathrm{~b}$ ). The coupling constant $\mathrm{g}_{\text {coll }}$ is measured between $70 \mathrm{mK}$ and $500 \mathrm{mK}$. The population for the lower Zeeman level scales as $N_{1} \sim N \frac{\exp (-x)}{\exp (-x)+\exp (x)}$ with $x=\hbar \omega / k_{b} T$ and $\mathrm{N}$ the total number of atoms. For the upper level we have $N_{2} \sim N \frac{\exp (x)}{\exp (-x)+\exp (x)}$. The collective coupling $g_{\text {coll }}(T)$, depends on the temperature through the relative difference of these populations, namely: $g_{\text {coll }}(T)=g_{c} \sqrt{N \tanh (x)}=g_{\text {coll }}(0) \sqrt{\tanh (x)}$. Fixing the resonance frequency, we obtain a collective coupling constant of $6.14 \mathrm{MHz}$ for site $2 \mathrm{a}$ extrapolating to zero temperature. From the fit we infer that the sample is highly polarized, reaching $90 \%$ polarization for the lowest temperatures in our experiment.

\section{CONCLUSIONS}

In conclusion, we have shown coupling between an $\mathrm{Er}^{3+}$ ensemble doped into a $\mathrm{Y}_{2} \mathrm{SiO}_{5}$ crystal and a superconducting CPW resonator, having a collective spin coupling of $4 \mathrm{MHz}$ and a spin linewidth of down to $75 \mathrm{MHz}$, 


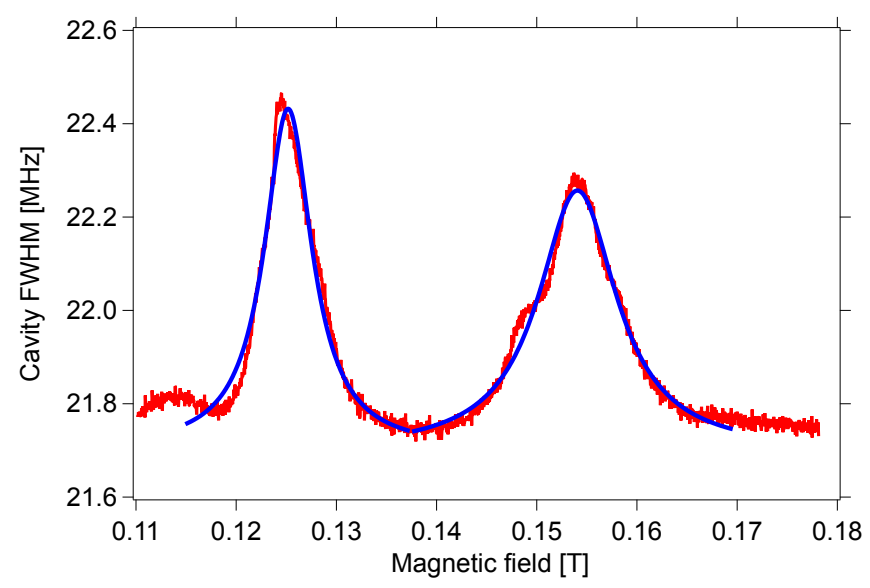

FIG. 3: Resonator linewidth as a function of magnetic field in the field region around $140 \mathrm{mT}$. Magnetic g-factors between 2 and 3 are extracted. Within each pair, the two lines represent spins aligned along different crystal axes that are related by symmetry. From the fit (solid lines, see text for details) we obtain a collective coupling rate of about $6 \mathrm{MHz}$ and a spin linewidth of about $100 \mathrm{MHz}$.

in good agreement with previous work ${ }^{30}$. The good coupling in the microwave regime, the widely used telecom transition and the good coherence properties show the potential for a coherent quantum optical - microwave interface made from an $\mathrm{Er}^{3+}$ ensemble doped into a $\mathrm{Y}_{2} \mathrm{SiO}_{5}$ crystal.

Though the collective enhancement effect of the spins participating leads to a coupling in the $\mathrm{MHz}$ range, we are not yet entering the strong coupling regime. The usage of a high-Q cavity should be advantageous to reach that goal. Moreover a narrower inhomogeneous linewidth could be obtained by reducing the $\mathrm{Er}^{3+}$ doping concentration. However, this will also imply less ion - spins participating, decreasing the coupling strength at the same time. Instead one could follow an interesting proposa ${ }^{45}$ and increase the coupling strength by further increasing the $\mathrm{Er}^{3+}$ doping concentration and enter a regime where the relaxation is governed by the single emitters properties taking advantage of so called "cavity protection."

For a future quantum-coherent interface, the low-field transition having a $\mathrm{g}$ - factor of 8.4 seems the most promising. Here a linewidth down to $75 \mathrm{MHz}$ is obtained and the moderate external magnetic field does not suppress superconductivity in the CPWR.

Instead of using the Zeeman splitting, one could use the hyperfine structure present in Kramers ions with nonzero nuclear magnetic spin (i.e. for Erbium $I=7 / 2$ ). The hyperfine interaction for Erbium is in the range of several hundred $\mathrm{MHz}$. This approach avoids the problem of inhomogeneity in the magnetic field, and transitions with low sensitivity to stray magnetic fields could be exploited. Although the properties of hyperfine transitions at zero or close to zero magnetic field has been less studied, we believe that this configuration, combined with the

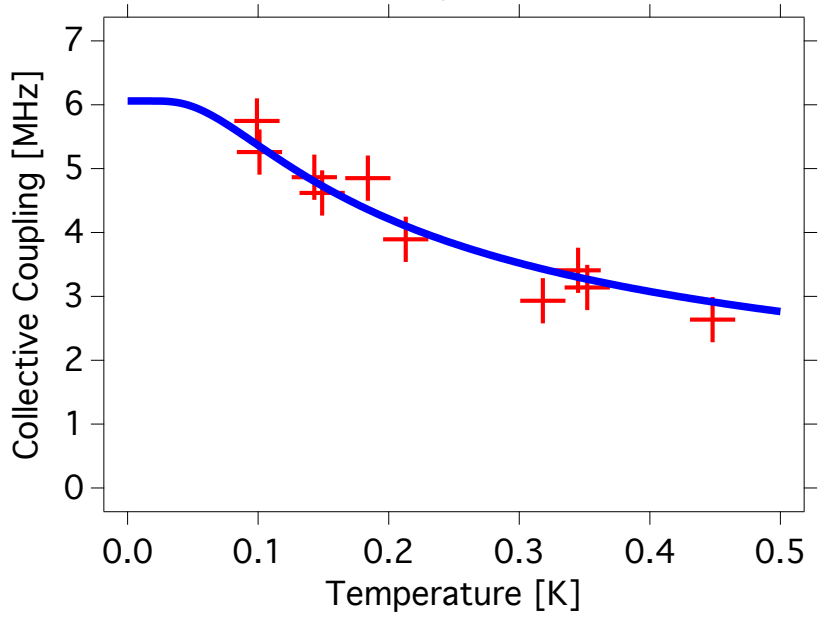

FIG. 4: Temperature dependence of the coupling for site 2 having a magnetic g-factor of 2.04 (transition labelled 2b). We can explain the dependence by assuming that the number of spins participating in the ensemble decreases as the spins are thermally depolarized (see text for details). We see that we reach $90 \%$ polarization at the lowest temperatures.

low-temperature conditions of circuit QED experiments, has advantages for the coherence properties of the spin ensemble.

\section{ACKNOWLEDGEMENT}

We would like to thank F. Persson and M. Pierre for experimental help. This work was supported by the EU integrated project SOLID. P.B. acknowledges the financial support through BMBF project QUIMP and RiSC grant of KIT and MWK of Baden-Wuerttemberg. M.S., N.S. and M.A. acknowledge financial support through EU integrated project Q-Essence.
* Electronic address: staudt@chalmers.se

1 H. J. Kimble, Nature 453, 1028 (2008).

2 M.A. Nielsen and I.L. Chuang, Quantum Computation and Quantum Information, Cambridge University Press, Cambridge, England (2000).

3 N. Gisin and R. Thew, Nature Photonics 1, 165 (2007).
${ }^{4}$ V. Giovannetti, S. Lloyd and L. Maccone, Science 306, 1330 (2004).

${ }^{5}$ I. Hoi, C. M. Wilson, G. Johansson, T. Palomaki, B. Peropadre, and P. Delsing, Phys. Rev. Lett. 107, 073601 (2011).

6 A. Wallraff, D. I. Schuster, A. Blais, L. Frunzio, R.-S. 
Huang, J. Majer, S. Kumar, S. M. Girvin, and R. J. Schoelkopf, Nature 431, 162 (2004).

7 C. M. Wilson, T. Duty, F. Persson, M. Sandberg, G. Johansson, P. Delsing, Phys. Rev. Lett. 98, 257003 (2007).

8 M. Sandberg, C. M. Wilson, F. Persson, T. Bauch, G. Johansson, V. Shumeiko, T. Duty, and P. Delsing, Appl. Phys. Lett. 92, 203501 (2008).

9 J. R. Johansson, G. Johansson, C. M. Wilson, F. Nori, Phys. Rev. Lett. 103, 147003 (2009).

10 C.M. Wilson, G. Johansson, T. Duty, F. Persson, M. Sandberg and P. Delsing, Phys. Rev. B 81, 024520 (2010).

11 F. Persson, C. M. Wilson, M. Sandberg, G. Johansson and P. Delsing, Nano Lett. 3, 953 (2010).

12 C.M. Wilson, T. Duty, M. Sandberg, F. Persson, V. Shumeiko, P. Delsing, P, Phys. Rev. Lett. 105, 233907 (2010).

13 C. M. Wilson, G. Johansson, A. Pourkabirian, M. Simoen, J. R. Johansson, T. Duty, F. Nori and P. Delsing, Nature 479, 376 (2011).

14 D. I. Schuster, A. A. Houck, J. A. Schreier, A. Wallraff, J. M. Gambetta, A. Blais, L. Frunzio, J. Majer, B. Johnson, M. H. Devoret, S. M. Girvin, and R. J. Schoelkopf, Nature 445, 515 (2007).

15 A. A. Houck, D. I. Schuster, J. M. Gambetta, J. A. Schreier, B. R. Johnson, J. M. Chow, L. Frunzio, J. Majer, M. H. Devoret, S. M. Girvin, and R. J. Schoelkopf, Nature 449, 328 (2007).

16 J. Majer, J. M. Chow, J. M. Gambetta, J. Koch, B. R. Johnson, J. A. Schreier, L. Frunzio, D. I. Schuster, A. A. Houck, A. Wallraff, A. Blais, M. H. Devoret, S. M. Girvin, and R. J. Schoelkopf, Nature 449, 443 (2007).

17 L. DiCarlo, M. D. Reed, L. Sun, B. R. Johnson, J. M. Chow, J. M. Gambetta, L. Frunzio, S. M. Girvin, M. H. Devoret and R. J. Schoelkopf, Nature 467, 574 (2011).

18 M. Neeley, R. C. Bialczak, M. Lenander, E. Lucero, M. Mariantoni, A. D. OConnell, D. Sank, H. Wang, M. Weides, J. Wenner, Y. Yin, T. Yamamoto, A. N. Cleland and J. M. Martinis, Nature 467, 570 (2011).

19 H. Paik, D. I. Schuster, L. S. Bishop, G. Kirchmair, G. Catelani, A. P. Sears, B. R. Johnson, M. J. Reagor, L. Frunzio, L. I. Glazman, S. M. Girvin, M. H. Devoret, and R. J. Schoelkopf, Phys. Rev. Lett. 107, 240501 (2011).

20 A. .S. Sørensen, C. H. van der Wal, L. I. Childress, M. D. Lukin, Phys. Rev. Lett. 92, 063601 (2004).

21 A. Imamoğlu, Phys. Rev. Lett. 102, 083602 (2009).

22 J. Verdú, H. Zoubi, Ch. Koller, J. Majer, H. Ritsch, and J. Schmiedmayer, Phys. Rev. Lett 103, 043603 (2009).

23 M. Wallquist, K. Hammerer, P. Rabl, M. Lukin, and P Zoller, Phys. Scr. T 137, 014001 (2009).

24 A. André, D. DeMille, J. M. Doyle, M. D. Lukin, S. E. Maxwell, P. Rabl, R. J. Schoelkopf and P. Zoller, Nature Physics 2, 636 (2006).

${ }^{25}$ R. J. Schoelkopf and S. M. Girvin, Nature 451, 664 (2008).

${ }^{26}$ Y. Kubo, F. R. Ong, P. Bertet, D. Vion, V. Jacques, D. Zheng, A. Dréau, J.-F. Roch, A. Auffeves, F. Jelezko, J. Wrachtrup, M.F. Barthe, P. Bergonzo, D. Esteve, Phys. Rev. Lett. 105, 140502 (2010)

27 D. I. Schuster, A. P. Sears, E. Ginossar, L. DiCarlo, L. Frunzio, J.J.L.Morton, H. Wu, G.A.D. Briggs, B.B. Buckley, D.D. Awschalom, R. J. Schoelkopf, Phys. Rev. Lett. 105, 140501 (2010).
${ }^{28}$ Y. Kubo, C. Grezes, A. Dewes, T. Umeda, J. Isoya, H. Sumiya, N. Morishita, H. Abe, S. Onoda, T. Ohshima, V. Jacques, A. Dréau, J.-F. Roch, I. Diniz, A. Auffeves, D. Vion, D. Esteve, P. Bertet, Phys. Rev. Lett. 107, 220501 (2011).

29 R. Amsuss, C. Koller, T. Nobauer, S. Putz, S. Rotter, K. Sandner, S. Schneider, M. Schrambock, G. Steinhauser, H. Ritsch, J. Schmiedmayer, J. Majer, Phys. Rev. Lett. 107, 060502 (2011).

${ }^{30}$ P. Bushev, A. K. Feofanov, H. Rotzinger, I. Protopopov, J. H. Cole, C. M. Wilson, G. Fischer, A. Lukashenko, and A. V. Ustinov, Phys. Rev. B. 84, 060501 (2011).

31 B. Lauritzen, J. Minar, H. de Riedmatten, M. Afzelius, N. Sangouard, C. Simon, and N.Gisin, Phys. Rev. Lett. 104, 080502 (2010).

32 J. Hegarty, M. M. Broer, B. Golding, J. R. Simpson, and J. B. MacChesney, Phys. Rev. Lett. 51, 2033 (1983).

${ }^{33}$ M. U. Staudt, S. R. Hastings-Simon, M. Afzelius, D. Jaccard, W. Tittel, N. Gisin, Optics Communications 266, 720 (2006).

34 R. M. Macfarlane, Journal of Luminescence 100, 1 (2002).

35 W. Tittel, M. Afzelius, T. Chanelière, R. L. Cone, S. Kröll, S. A. Moiseev and M. Sellars, Laser and Photonics Reviews 4, 244 (2010).

36 N. Sangouard, C. Simon, H. de Riedmatten and N. Gisin, Rev. Mod. Phys. 83, 3 (2011).

37 J.J. Longdell and M.J. Sellars, Phys. Rev. A 69, 032307 (2004).

38 L. Rippe, B. Julsgaard, A. Walther, Y. Ying, S. Kröll, Phys. Rev. A 77, 022307 (2008).

39 M. Sabooni,F. Beaudoin, A. Walther, N. Lin, A. Amari, M. Huang, S. Kröll, Phys. Rev. Lett. 105, 060501 (2010).

40 M. P. Hedges, J.J. Longdell, Y. Li and M.J. Sellars, Nature 465, 1052 (2010).

41 E. Saglamyurek, N. Sinclair, J. Jin, J. A. Slater, D. Oblak, F. Bussières, M. George, R. Ricken, W. Sohler and W. Tittel, Nature 469, 512 (2011).

42 C. Clausen, I. Usmani, F. Bussières, N. Sangouard, M. Afzelius, H. de Riedmatten, N. Gisin, Nature 469, 508 (2011).

43 V. Damon, M. Bonarota, A. Louchet-Chauvet, T. Chanelière, J.-L. Le Gouët, New Journal of Physics, 13 093031 (2011).

44 O. Guillot-Noël, Ph. Goldner, Y. Le Du, E. Baldit, P.Monnier, and K. Bencheikh, Phys. Rev. B 74, 214409 (2006).

45 I. Diniz, S. Portolan, R. Ferreira, J.M. Gerard, P. Bertet, and A. Auffeves, Phys. Rev. A 84, 063810 (2011).

46 Y. Sun, T. Böttger, C. W. Thiel, R. L. Cone, Phys. Rev. B 77, 085124 (2008).

47 T. Böttger, PhD thesis, Montana State University, Bozeman, Montana, USA (2002).

48 D. I. Schuster, A.P. Sears, E. Ginossar, L. DiCarlo, L. Frunzio, J.J.L. Morton, H. Wu, G.A.D. Briggs, B.B. Buckley, D.D. Awschalom, R.J. Schoelkopf, Phys. Rev. Lett., 105140501 (2011).

49 The hyperfine site peaks are detuned by: $\Delta_{\text {hypert }}=$ $\omega_{r}-g_{l} \mu_{b}\left(B \pm A_{0} M_{I}\right) / \hbar$ where $\mathrm{A}_{0}$ is the hyperfine coupling constant and $\mathrm{M}_{I}$ is the nuclear spin projection. 